\title{
Safe Provision of Systemic Anti-Cancer Treatment for Urological Cancer Patients During COVID-19: A Tertiary Centre Experience in the First Wave of COVID-19
}

Alfred Chung Pui So

Guy's and St Thomas' NHS Foundation Trust

Christina Karampera

Guy's and St Thomas' NHS Foundation Trust

Muhammad Khan

Guy's and St Thomas' NHS Foundation Trust

Beth Russell

King's College London

Charlotte Moss

King's College London

Maria J Monroy-Iglesias

King's College London

Kiruthikah Thillai

Guy's and St Thomas' NHS Foundation Trust

Debra Hannah Josephs

Guy's and St Thomas' NHS Foundation Trust

\section{Elias Pintus}

Guy's and St Thomas' NHS Foundation Trust

\section{Sarah Rudman}

Guy's and St Thomas' NHS Foundation Trust

Mieke Hemelrijck

King's College London

Saoirse Dolly

Guy's and St Thomas' NHS Foundation Trust

Deborah Enting ( $\square$ deborah.enting@gstt.nhs.uk)

Guy's and St Thomas' NHS Foundation Trust

\section{Research Article}

Keywords: urological cancer, patients, COVID-19, wave 
Posted Date: January 10th, 2022

DOI: https://doi.org/10.21203/rs.3.rs-1099202/v1

License: (c) (1) This work is licensed under a Creative Commons Attribution 4.0 International License. Read Full License 


\section{Abstract \\ Background}

Safe provision of systemic anti-cancer treatment (SACT) during the COVID-19 pandemic remains an ongoing concern amongst clinicians.

\section{Methods}

Retrospective analysis on uro-oncology patients who continued or started SACT between 1st March and 31st May 2020 during the pandemic (with 2019 as a comparator).

\section{Results}

441 patients received SACT in 2020 (292 prostate, 101 renal, 38 urothelial, 10 testicular) compared to 518 patients in 2019 (340 prostate, 121 renal, 42 urothelial, 15 testicular). In 2020, there were $75.00 \%$ fewer patients with stage 3 cancers receiving SACT $(p<0.0001)$ and $94.44 \%$ fewer patients receiving radical treatment $(p=0.0019)$. The number of patients started on a new line of SACT was similar between both years (118 in 2019 vs 102 in 2020; $p=N$.S) but with 53.45\% fewer patients started on chemotherapy in 2020 $(p=0.00067)$. Overall, 5 patients tested positive for COVID-19 (one asymptomatic, two moderate pneumonitis, one severe pneumonitis). Compared to 2019 , 30-day mortality was similar ( $1.69 \%$ in 2019 vs $0.98 \%$ in $2020 ; p=N . S)$ whereas the 6 -month mortality was lower $(9.32 \%$ in 2019 vs $1.96 \%$ in $2020 ; p=0.023$ ) in 2020 .

\section{Conclusion}

This single-centre study demonstrated that uro-oncology patients can safely receive SACT during COVID-19 pandemic with a low incidence of infection and mortality.

\subsection{Background}

On the 23rd March, 2020, the UK government officially announced the first national lockdown in response to the COVID-19 pandemic. Over the past year and a half we have seen dramatic changes in healthcare service provision, repurposing of drugs for severe COVID-19 infections, and the arrival of COVID-19 vaccines. Coming out of the end of the second lockdown with cautious anticipation of a third wave, we need to address ongoing concerns on how to deliver essential non-COVID-19 services to the public.

All aspects of cancer services have been significantly impacted by the pandemic, resulting in a backlog of patients who may present with more aggressive disease or in extremis. During the early stages of the pandemic, initial observational studies suggested that having active cancer and receiving systemic anticancer treatments (SACT) increased a patient's risk of severe COVID-19 and subsequent death ${ }^{1-3}$. Since 
then, there has been significant increase in published material evaluating this risk in cancer patients. Although the data remains heterogenous between studies due to variations in oncological characteristics, comorbidities, and study design, common risk factors for poor outcomes from COVID-19 include active cancer (especially haematological malignancies) and those similar to the general population (e.g age, male sex, cardiovascular comorbidities) ${ }^{4-11}$. In addition, receiving SACT does not appear to be a risk factor for severe COVID-19 and death ${ }^{6-12}$. However, the relationship between SACT and COVID-19 outcomes is complex and requires ongoing targeted studies due to significant variations in chemotherapy regimens for different tumour groups.

Whilst the national institute for health and care excellence (NICE) and international experts have provided recommendations on the prioritisation of cancer treatments, the ability to deliver these will vary depending on resources available to the healthcare provider. Our tertiary cancer centre in South London treats around 8,800 patients per annum (of which 4,500 are new patients) and is one of the largest comprehensive cancer centres in the UK. Our institute was also at the epicentre of the COVID-19 pandemic with London having the highest rates of infection and COVID-19-related deaths. Here, we report our experience in delivering SACT to patients with urological cancers during the first wave in order to support clinicians in developing guidelines for managing these patients throughout the pandemic.

\subsection{Methods}

\subsection{Restructuring cancer services at Guy's Cancer Centre}

During the first wave of COVID-19 there were several key changes in how we restructured our cancer services in order to provide essential cancer services whilst minimising the risk of COVID-19 (Table 1). 
Table 1

Strategies to reduce risk of COVID-19 transmission at our hospital.

\begin{tabular}{|c|c|}
\hline Variable & Strategy \\
\hline \multirow{9}{*}{$\begin{array}{l}\text { Limiting } \\
\text { transmission } \\
\text { risk }\end{array}$} & - Testing patients receiving SACT at the cancer centre \\
\hline & $\begin{array}{l}\text { o Staff members were deployed to the front of hospital to ask COVID-19 screening } \\
\text { questions and check patient temperatures prior to entering. }\end{array}$ \\
\hline & $\begin{array}{l}\text { o Patients who tested positive for COVID- } 19 \text { but required an in-person review or were } \\
\text { suitable to continue SACT followed a specific COVID- } 19 \text { pathway within the Cancer } \\
\text { Centre (separated from other patients). Patients receiving SACT were treated in a side } \\
\text { room by dedicated nursing staff. }\end{array}$ \\
\hline & - Visitors \\
\hline & $\begin{array}{l}\text { o Majority of visitors and relatives were not allowed to attend the hospital with the } \\
\text { patients. However, there were several extenuating circumstances including patients } \\
\text { receiving end-of-life care and vulnerable individuals. }\end{array}$ \\
\hline & - Staff members \\
\hline & $\begin{array}{l}\text { o Staff members conducted basic measures to reduce risk of transmission including } \\
\text { hand hygiene, wearing appropriate PPE depending on the clinical context, social } \\
\text { distancing, and self-isolation if they develop symptoms suggestive of COVID-19. }\end{array}$ \\
\hline & - Social distancing \\
\hline & $\begin{array}{l}\text { o Infection control teams helped determine the limit in which the number of people can } \\
\text { be in a room, elevator, or waiting area. }\end{array}$ \\
\hline \multirow[t]{5}{*}{ Consultations } & - Virtual and telephone consultants \\
\hline & $\begin{array}{l}\text { o There has been significant increase in utilising technology to aid virtual and } \\
\text { telephone consultations, limiting the number of potential contacts both the patient and } \\
\text { clinician will have. This also allowed ongoing communication with the patient and their } \\
\text { relatives during uncertain times throughout the pandemic. }\end{array}$ \\
\hline & $\begin{array}{l}\text { o Although this may not be appropriate for all circumstances, this was particularly } \\
\text { useful for patients well established on treatments or at a particularly high risk of severe } \\
\text { COVID-19 due to comorbidities. }\end{array}$ \\
\hline & - Deferring follow-up consultations \\
\hline & $\begin{array}{l}\text { o We extended the duration between follow-up consultations for some patients who } \\
\text { were established on their current treatment. This also applied to certain routine follow- } \\
\text { up imaging in which there was a low chance that it will impact the current treatment } \\
\text { regimen. }\end{array}$ \\
\hline
\end{tabular}




\begin{tabular}{|c|c|}
\hline Variable & Strategy \\
\hline \multirow{5}{*}{$\begin{array}{l}\text { Outsourcing } \\
\text { services }\end{array}$} & - Satellite hubs \\
\hline & $\begin{array}{l}\text { o These were implemented with the aid of ambulance services to provide mobile blood } \\
\text { testing facilities. These tests were then sent to the centre and reviewed by the clinical } \\
\text { staff. }\end{array}$ \\
\hline & $\begin{array}{l}\text { o This allowed patients to limit their duration of travel, avoid public transports, and } \\
\text { limit contact with others at the cancer centre. }\end{array}$ \\
\hline & - Courier services \\
\hline & $\begin{array}{l}\text { o Patients that are established on SACT can have their medications couriered to their } \\
\text { home rather than pick it up at the cancer centre. }\end{array}$ \\
\hline $\begin{array}{l}\text { Treatment } \\
\text { prioritisation }\end{array}$ & $\begin{array}{l}\text { - With recommendations from NICE and expert consensus, treatments were prioritised } \\
\text { based on risk-benefit to contracting COVID- } 19 \text {, probability of cure, reducing } \\
\text { immunosuppressive states, and availability of resources to deliver these services. } \\
\text { These decisions were discussed with the patient and were considered on a case-by- } \\
\text { case scenario. }\end{array}$ \\
\hline
\end{tabular}

\subsection{Data analysis}

All patients at a tertiary cancer centre in London receiving at least one SACT cycle for a urological cancer between 1st March, 2020 and 31st May, 2020 were included for data analysis. We used the same timeframe in 2019 as a comparator group. Patient demographics, oncological characteristics, SACT information, and COVID-19 status were extracted using our patient electronic records and chemotherapy prescribing system. Socioeconomic status was categorised into low, middle, and high using the English Indices of Multiple Deprivation (IMD) ranking based on postcodes. Cancers were staged according to the UICC 8th staging system. COVID-19 infection was defined as a positive RT-PCR test. Patients with only radiological changes or symptoms suggestive of COVID-19 without a positive RT-PCR test were excluded. COVID-19 positive patients were then categorised into asymptomatic, mild, moderate, or severe disease as defined by the WHO criteria ${ }^{13}$. Chi-square testing was used to compare demographics and clinical characteristics in 2020 with 2019. A $p$-value of 0.05 was considered statistically significant. Subsequent post-hoc subgroup analysis based on a statistically significant Chi-square test was performed using the Bonferroni correction for multiple comparisons. All data was collected as part of Guy's Cancer Cohort (Ethics Reference number: $18 / \mathrm{NW} / 0297)^{14}$.

\subsection{Results}

In the study period, there was a total of 441 patients who received SACT in 2020 (292 prostate, 101 renal, 38 urothelial, 10 testicular) compared to 518 patients in 2019 (340 prostate, 121 renal, 42 urothelial, 15 testicular) with an overall decline of $14.86 \%(p=N . S)$ (Table 2). Overall, there was a reduction in the number of patients receiving SACT in 2020 during the first wave of the COVID-19 pandemic with the largest reductions seen in prostate $(14.12 \%)$ and renal cancers $(16.53 \%)$. There were no significant differences in patient demographics with regards to age, sex, socioeconomic status, and ethnicity (Table 3, Table S1). 
There was a significant amount of missing data on patient performance status in $2020(26.35 \% ; p<0.0001)$ which made it difficult to comment on any differences. 
Table 2

Oncological characteristics of patients receiving SACT.

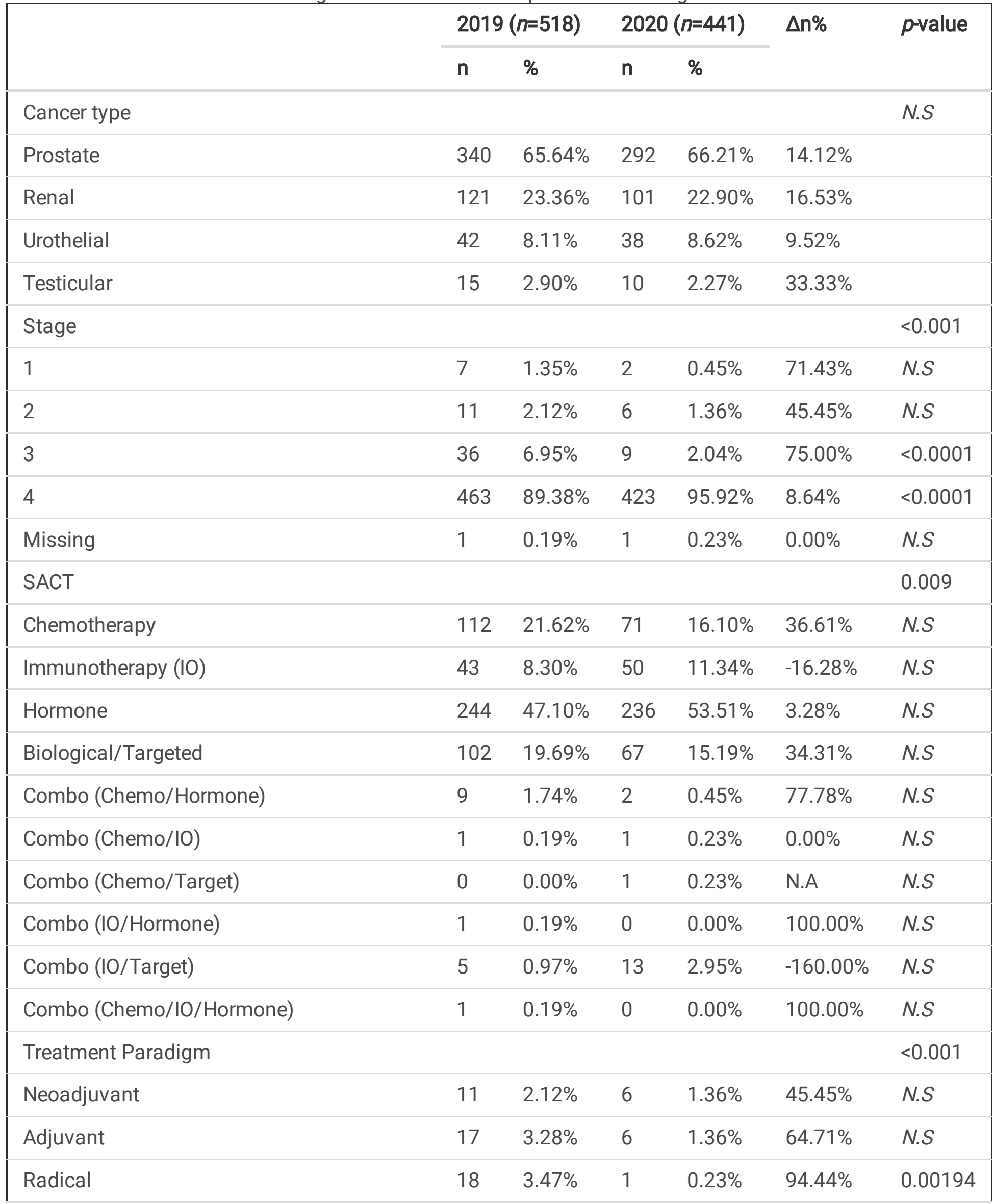




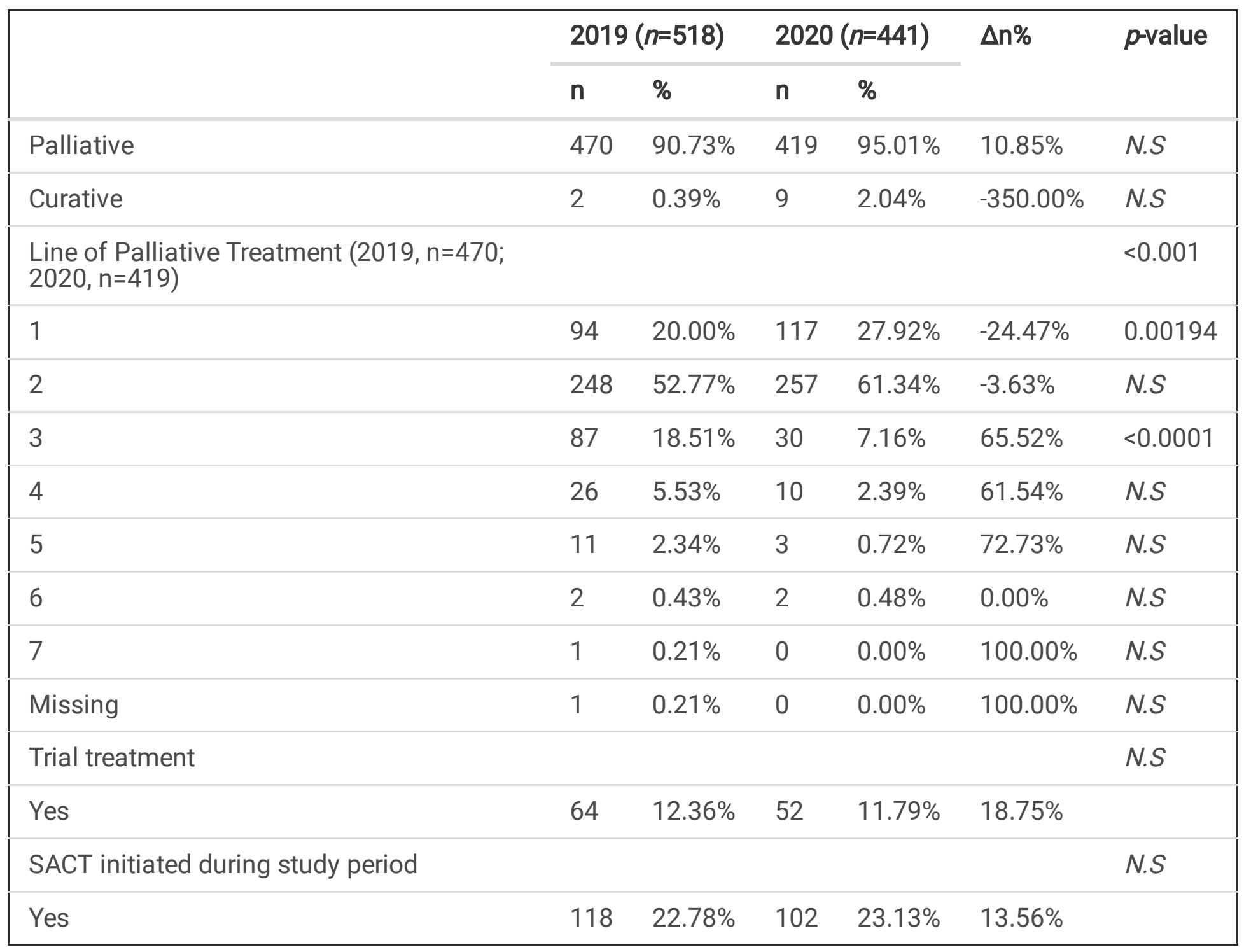


Table 3

Patient demographics.

\begin{tabular}{|c|c|c|c|c|c|c|}
\hline & $2019(n=51$ & & $2020(n=44$ & & $\Delta \mathrm{n} \%$ & $p$-value \\
\hline & $\mathbf{n}$ & $\%$ & $\mathrm{n}$ & $\%$ & & \\
\hline Sex & & & & & & N.S \\
\hline Male & 467 & $90.15 \%$ & 402 & $91.16 \%$ & $13.92 \%$ & \\
\hline Female & 51 & $9.85 \%$ & 39 & $8.84 \%$ & $23.53 \%$ & \\
\hline Age & & & & & & N.S \\
\hline$<50$ & 26 & $5.02 \%$ & 24 & $5.44 \%$ & $7.69 \%$ & \\
\hline $50-59$ & 62 & $11.97 \%$ & 49 & $11.11 \%$ & $20.97 \%$ & \\
\hline $60-69$ & 164 & $31.66 \%$ & 152 & $34.47 \%$ & $7.32 \%$ & \\
\hline $70-79$ & 178 & $34.36 \%$ & 139 & $31.52 \%$ & $21.91 \%$ & \\
\hline$\geq 80$ & 88 & $16.99 \%$ & 77 & $17.46 \%$ & $12.50 \%$ & \\
\hline Mean (SD) - years & $68.8(11.5)$ & - & $68.6(11.3)$ & - & - & \\
\hline Socioeconomic status (IMD) & & & & & & N.S \\
\hline Low & 168 & $32.43 \%$ & 145 & $32.88 \%$ & $13.69 \%$ & \\
\hline Middle & 170 & $32.82 \%$ & 143 & $32.43 \%$ & $15.88 \%$ & \\
\hline High & 178 & $34.36 \%$ & 152 & $34.47 \%$ & $14.61 \%$ & \\
\hline Missing & 2 & $0.39 \%$ & 1 & $0.23 \%$ & $50.00 \%$ & \\
\hline Ethnicity & & & & & & N.S \\
\hline White British & 239 & $46.14 \%$ & 190 & $43.08 \%$ & $20.50 \%$ & \\
\hline White Other & 26 & $5.02 \%$ & 28 & $6.35 \%$ & $-7.69 \%$ & \\
\hline Black Caribbean & 36 & $6.95 \%$ & 21 & $4.76 \%$ & $41.67 \%$ & \\
\hline Black African & 17 & $3.28 \%$ & 19 & $4.31 \%$ & $-11.76 \%$ & \\
\hline Black Other & 4 & $0.77 \%$ & 5 & $1.13 \%$ & $-25.00 \%$ & \\
\hline Asian & 13 & $2.51 \%$ & 7 & $1.59 \%$ & $46.15 \%$ & \\
\hline Mixed & 1 & $0.19 \%$ & 0 & $0.00 \%$ & $100.00 \%$ & \\
\hline Other & 8 & $1.54 \%$ & 7 & $1.59 \%$ & $12.50 \%$ & \\
\hline Unknown & 174 & $33.59 \%$ & 164 & $37.19 \%$ & $5.75 \%$ & \\
\hline Performance status (ECOG) & & & & & & $<0.001$ \\
\hline
\end{tabular}




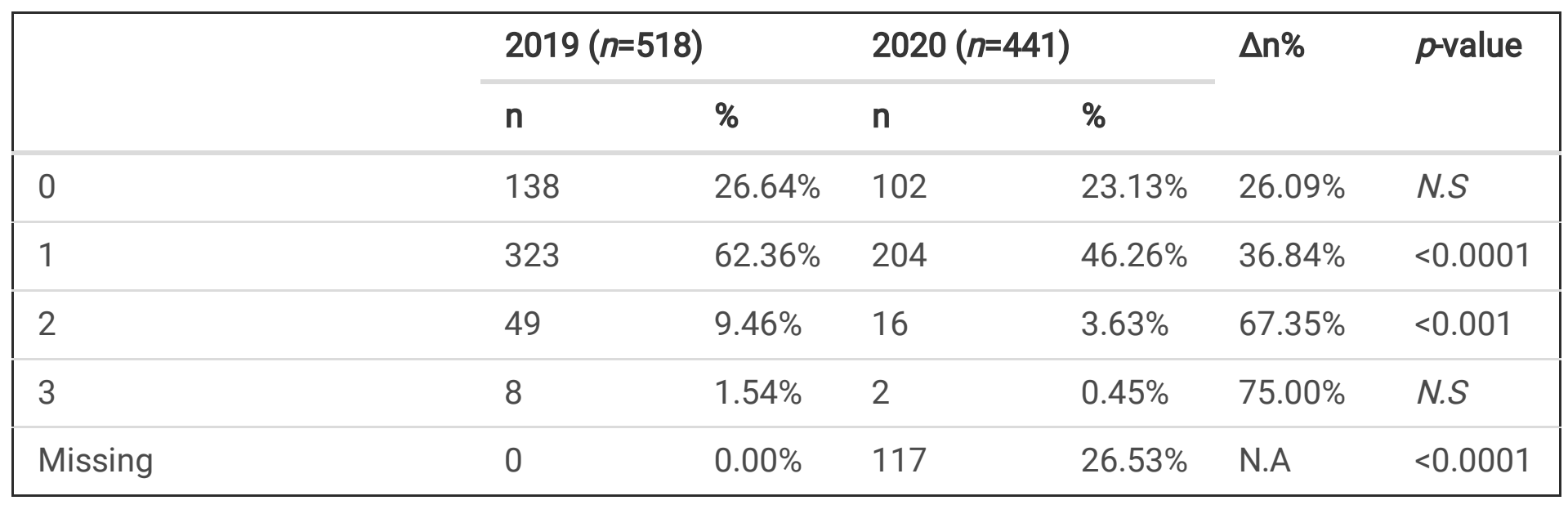

The majority of the patients had advanced or metastatic cancers (stage 3-4). There was a greater decline in the proportion of patients who received SACT with stage 3 cancers $(75.00 \% ; p<0.0001)$ compared to stage 4 cancers $(8.42 \% ; p<0.0001)$ in 2020 . This difference was best observed with prostate cancer where there were 18 fewer patients with stage 3 cancers who received SACT in 2020 (20 vs 2; $p<0.0001$ ) (Table S2).

Hormone treatment was the most common type of SACT delivered followed by chemotherapy, targeted therapy, and immunotherapy in both 2019 and 2020. The largest reductions were seen with chemotherapy $(36.61 \% ; p=N . S)$ and targeted therapy $(34.31 \% ; p=N . S)$. This was particularly evident in renal cancers with a decline of $42.71 \%$ in the number of patients receiving targeted therapy ( $p=0.00067)$ (Table S2). Furthermore, there was a small increase in the number of patients with renal cancer receiving immunotherapy alone and in combination with targeted treatment. In the prostate cancer group, whilst there was a reduction in number of patients receiving chemotherapy in $2020(32.91 \% ; p=N$.S ), there was no significant difference in the number of patients receiving hormone therapy (Table S2). The majority of the prostate cancer group receiving hormone therapy were treated with novel hormone agents (i.e abiraterone, enzalutamide) in both 2019 (94.26\%) and 2020 (91.95\%) (Table S2). Unfortunately, due to the pandemic there were generally fewer urological patients receiving SACT as part of a radical regimen (from 3.47-0.23\%; $p=0.0019$ ) and fewer patients going onto 3rd line palliative SACT (from 16.80-6.80\%; $p<0.0001$ ) (Table 2). The number of patients on clinical trial treatments were similar between 2019 and 2020 (64 vs 52; $p=N . S$ ).

The number of patients that were started on a new line of SACT was similar between both years (118 vs $102 ; p=N . S$ ). However, further subgroup analysis suggests that there were less prostate cancer patients being started on SACT (74 vs $57 ; p=N . S$ ) and less patients with urological cancers started on chemotherapy (58 vs $27 ; p=0.00067$ ) in 2020 (Table 4). The type of patients that were started on SACT during COVID-19 were generally younger with a performance status between $0-1$. The majority received palliative SACT and had similar number of lines of palliative treatment. Fewer patients with stage 1 disease (primarily testicular cancers) were started on SACT and fewer patients received adjuvant SACT. The number of patients starting on curative or radical treatments were similar between both years. In patients started on SACT during COVID19, the 30-day mortality was similar (1.69\% vs $0.98 \% ; p=N$.S) compared with 2019 (Table 4). On the other hand, the 6-month mortality was lower in 2020 (9.32\% vs $1.96 \% ; p=0.023)$. 
Table 4

Patient demographics and oncological characteristics of patients started on SACT between 1st March to 31 st May in 2020 during COVID-19 (with 2019 as a comparator).

\begin{tabular}{|c|c|c|c|c|c|c|}
\hline & \multicolumn{2}{|c|}{$2019(n=118)$} & \multicolumn{2}{|l|}{$2020(n=102)$} & \multirow[t]{2}{*}{$\Delta \mathrm{n} \%$} & \multirow[t]{2}{*}{$p$-value } \\
\hline & $\mathbf{n}$ & $\%$ & $\mathbf{n}$ & $\%$ & & \\
\hline Sex & & & & & & N.S \\
\hline Male & 106 & $89.83 \%$ & 90 & $88.24 \%$ & $15.09 \%$ & \\
\hline Age & & & & & & N.S \\
\hline Mean (SD) - years & $\begin{array}{l}66.92 \\
(12.16)\end{array}$ & - & $65.12(13.23)$ & - & - & \\
\hline Socioeconomic status (IMD) & & & & & & N.S \\
\hline Low & 37 & $31.36 \%$ & 40 & $39.22 \%$ & $-8.11 \%$ & \\
\hline Middle & 43 & $36.44 \%$ & 28 & $27.45 \%$ & $34.88 \%$ & \\
\hline High & 38 & $32.20 \%$ & 33 & $32.35 \%$ & $13.16 \%$ & \\
\hline Missing & 0 & $0.00 \%$ & 1 & $0.98 \%$ & N.A & \\
\hline Ethnicity & & & & & & N.S \\
\hline White British & 44 & $37.29 \%$ & 29 & $28.43 \%$ & $34.09 \%$ & \\
\hline White Other & 9 & $7.63 \%$ & 9 & $8.82 \%$ & $0.00 \%$ & \\
\hline Black Caribbean & 9 & $7.63 \%$ & 5 & $4.90 \%$ & $44.44 \%$ & \\
\hline Black African & 3 & $2.54 \%$ & 4 & $3.92 \%$ & $-33.33 \%$ & \\
\hline Black Other & 0 & $0.00 \%$ & 1 & $0.98 \%$ & N.A & \\
\hline Asian & 1 & $0.85 \%$ & 0 & $0.00 \%$ & $100.00 \%$ & \\
\hline Mixed & 1 & $0.85 \%$ & 0 & $0.00 \%$ & $100.00 \%$ & \\
\hline Other & 1 & $0.85 \%$ & 1 & $0.98 \%$ & $0.00 \%$ & \\
\hline Unknown & 50 & $42.37 \%$ & 53 & $51.96 \%$ & $-6.00 \%$ & \\
\hline Performance status (ECOG) & & & & & & $<0.001$ \\
\hline 0 & 42 & $35.59 \%$ & 27 & $26.47 \%$ & $35.71 \%$ & N.S \\
\hline 1 & 62 & $52.54 \%$ & 61 & $59.80 \%$ & $1.61 \%$ & N.S \\
\hline 2 & 13 & $11.02 \%$ & 3 & $2.94 \%$ & $76.92 \%$ & N.S \\
\hline 3 & 1 & $0.85 \%$ & 0 & $0.00 \%$ & $100.00 \%$ & N.S \\
\hline Missing & 0 & $0.00 \%$ & 11 & $10.78 \%$ & N.A & 0.00022 \\
\hline
\end{tabular}




\begin{tabular}{|c|c|c|c|c|c|c|}
\hline & \multicolumn{2}{|c|}{$2019(n=118)$} & \multicolumn{2}{|c|}{$2020(n=102)$} & \multirow[t]{2}{*}{$\Delta \mathrm{n} \%$} & \multirow[t]{2}{*}{$p$-value } \\
\hline & $\mathrm{n}$ & $\%$ & $\mathrm{n}$ & $\%$ & & \\
\hline Cancer type & & & & & & N.S \\
\hline Prostate & 74 & $62.71 \%$ & 57 & $55.88 \%$ & $22.97 \%$ & \\
\hline Renal & 22 & $18.64 \%$ & 21 & $20.59 \%$ & $4.55 \%$ & \\
\hline Urothelial & 14 & $11.86 \%$ & 17 & $16.67 \%$ & $-21.43 \%$ & \\
\hline Testicular & 8 & $6.78 \%$ & 7 & $6.86 \%$ & $12.50 \%$ & \\
\hline Stage & & & & & & N.S \\
\hline 1 & 7 & $5.93 \%$ & 1 & $0.98 \%$ & $85.71 \%$ & \\
\hline 2 & 4 & $3.39 \%$ & 5 & $4.90 \%$ & $-25.00 \%$ & \\
\hline 3 & 7 & $5.93 \%$ & 4 & $3.92 \%$ & $42.86 \%$ & \\
\hline 4 & 100 & $84.75 \%$ & 92 & $90.20 \%$ & $8.00 \%$ & \\
\hline Missing & 0 & $0.00 \%$ & 0 & $0.00 \%$ & N.A & \\
\hline SACT & & & & & & 0.012 \\
\hline Chemotherapy & 58 & $49.15 \%$ & 27 & $26.47 \%$ & $53.45 \%$ & 0.00067 \\
\hline Immunotherapy & 15 & $12.71 \%$ & 18 & $17.65 \%$ & $-20.00 \%$ & N.S \\
\hline PD-1/L1 & 11 & $9.32 \%$ & 15 & $14.71 \%$ & $-36.36 \%$ & \\
\hline PD-1/L1 + CTLA-4 & 3 & $2.54 \%$ & 4 & $3.92 \%$ & $-33.33 \%$ & \\
\hline Vaccine & 1 & $0.85 \%$ & 0 & $0.00 \%$ & $100.00 \%$ & \\
\hline Hormone & 33 & $27.97 \%$ & 43 & $42.16 \%$ & $-30.30 \%$ & N.S \\
\hline Novel hormone agents & 33 & $27.97 \%$ & 41 & $40.20 \%$ & $-24.24 \%$ & \\
\hline Biological/Targeted & 11 & $9.32 \%$ & 12 & $11.76 \%$ & $-9.09 \%$ & N.S \\
\hline Combo (Chemo/Hormone) & 1 & $0.85 \%$ & 1 & $0.98 \%$ & $0.00 \%$ & N.S \\
\hline Combo (IO/Target) & 0 & $0.00 \%$ & 1 & $0.98 \%$ & N.A & N.S \\
\hline Treatment Paradigm & & & & & & 0.005 \\
\hline Neoadjuvant & 8 & $6.78 \%$ & 4 & $3.92 \%$ & $50.00 \%$ & N.S \\
\hline Adjuvant & 10 & $8.47 \%$ & 2 & $1.96 \%$ & $80.00 \%$ & N.S \\
\hline Radical & 4 & $3.39 \%$ & 0 & $0.00 \%$ & $100.00 \%$ & N.S \\
\hline Palliative & 95 & $80.51 \%$ & 90 & $88.24 \%$ & $5.26 \%$ & N.S \\
\hline Curative & 1 & $0.85 \%$ & 6 & $5.88 \%$ & $-500.00 \%$ & N.S \\
\hline
\end{tabular}




\begin{tabular}{|c|c|c|c|c|c|c|c|}
\hline & \multicolumn{2}{|c|}{$2019(n=118)$} & \multicolumn{3}{|c|}{$2020(n=102)$} & \multirow[t]{2}{*}{$\Delta n \%$} & \multirow[t]{2}{*}{$p$-value } \\
\hline & $n$ & $\%$ & $n$ & & $\%$ & & \\
\hline $\begin{array}{l}\text { Line of Palliative Treatment } \\
(2019, n=95 ; 2020, n=90)\end{array}$ & & & & & & & $<0.001$ \\
\hline 1 & 24 & $20.34 \%$ & & 28 & $27.45 \%$ & $-16.67 \%$ & 0.0019 \\
\hline 2 & 49 & $41.53 \%$ & & 50 & $49.02 \%$ & $-2.04 \%$ & N.S \\
\hline 3 & 14 & $11.86 \%$ & & 8 & $7.84 \%$ & $42.86 \%$ & $<0.0001$ \\
\hline 4 & 5 & $4.24 \%$ & & 2 & $1.96 \%$ & $60.00 \%$ & N.S \\
\hline 5 & 2 & $1.69 \%$ & & 1 & $0.98 \%$ & $50.00 \%$ & N.S \\
\hline 6 & 0 & $0.00 \%$ & & 1 & $0.98 \%$ & N.A & N.S \\
\hline 7 & 1 & $0.85 \%$ & & 0 & $0.00 \%$ & $100.00 \%$ & N.S \\
\hline Trial treatment & & & & & & & N.S \\
\hline Yes & 7 & $5.93 \%$ & & 3 & $2.94 \%$ & $57.14 \%$ & \\
\hline \multirow[t]{2}{*}{ 30-day mortality } & & & & & & & N.S \\
\hline & 2 & $1.69 \%$ & 1 & & $0.98 \%$ & $50.00 \%$ & \\
\hline \multirow[t]{2}{*}{ 6-month mortality } & & & & & & & 0.023 \\
\hline & 11 & $9.32 \%$ & 2 & & $1.96 \%$ & $81.82 \%$ & \\
\hline
\end{tabular}

Of the 441 patients who received SACT during the study period, 5 tested positive for COVID-19 (2 prostate, 2 renal, 1 bladder) (Table 5). All patients were male, $\geq 60$ years of age, had stage 4 urological cancer and receiving palliative SACT ( 2 hormone, 2 targeted, 1 immunotherapy). In addition, 4 were from a lower socioeconomic background, 3 had more than one comorbidity, and 3 had polypharmacy. With regards to COVID-19 severity, 1 patient had asymptomatic infection, 2 had moderate COVID-19 pneumonitis, and 1 died from COVID-19. The patient who died had metastatic bladder cancer and died from severe COVID-19 pneumonitis with thromboembolic complications. 
Table 5

Patient demographics and oncological characteristics of patients tested positive for COVID-19.

\begin{tabular}{|c|c|c|}
\hline & \multicolumn{2}{|c|}{$2019(n=118)$} \\
\hline & $\mathbf{n}$ & $\%$ \\
\hline \multicolumn{3}{|l|}{ Patient demographics } \\
\hline \multicolumn{3}{|l|}{ Sex } \\
\hline Male & 5 & $100.00 \%$ \\
\hline \multicolumn{3}{|l|}{ Age } \\
\hline Mean (SD) - years & \multicolumn{2}{|c|}{$60.4(12.9)$} \\
\hline \multicolumn{3}{|c|}{ Socioeconomic status (IMD) } \\
\hline Low & 4 & $80.00 \%$ \\
\hline Missing & 1 & $20.00 \%$ \\
\hline \multicolumn{3}{|l|}{ Ethnicity } \\
\hline White British & 2 & $40.00 \%$ \\
\hline Black African & 1 & $20.00 \%$ \\
\hline Other & 1 & $20.00 \%$ \\
\hline Unknown & 1 & $20.00 \%$ \\
\hline \multicolumn{3}{|l|}{ Associated comorbidities } \\
\hline \multicolumn{3}{|l|}{ Comorbidities } \\
\hline Hypertension & 3 & $60.00 \%$ \\
\hline Diabetes & 3 & $60.00 \%$ \\
\hline Lung conditions & 0 & $0.00 \%$ \\
\hline Renal impairment & 1 & $20.00 \%$ \\
\hline Liver conditions & 0 & $0.00 \%$ \\
\hline Cerebrovascular disease & 0 & $0.00 \%$ \\
\hline Frailty & 1 & $20.00 \%$ \\
\hline Long-term steroid use & 0 & $0.00 \%$ \\
\hline \multicolumn{3}{|l|}{ Number of Comorbidities } \\
\hline 0 & 2 & $40.00 \%$ \\
\hline 1 & 0 & $0.00 \%$ \\
\hline
\end{tabular}




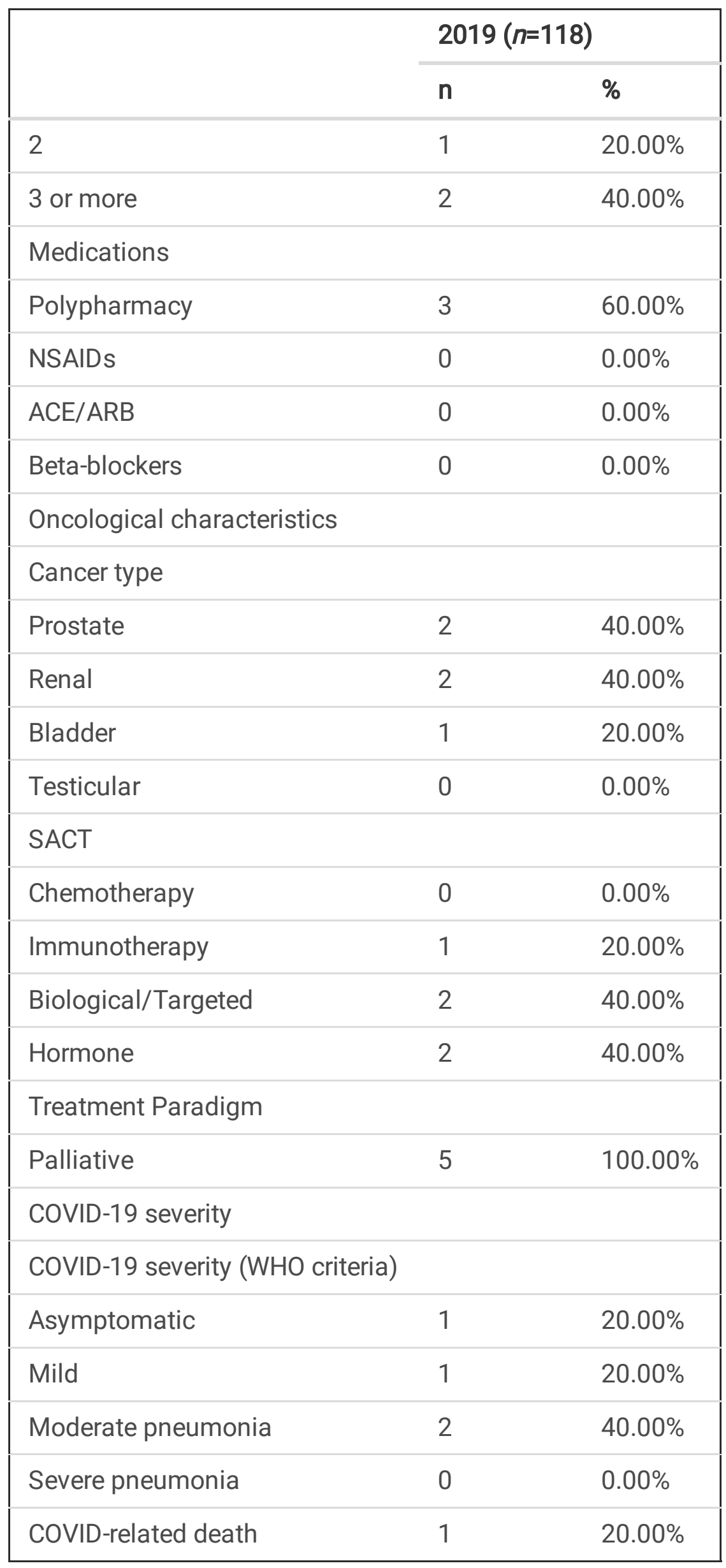




\subsection{Discussion}

In this single-centre retrospective cohort study, we report the outcomes of urological oncology patients receiving SACT during the first wave of the COVID-19 pandemic. Despite an inevitable decline in the number of patients receiving SACT during COVID-19, we were able to provide a safe high-quality urological cancer SACT pathway with a low incidence of COVID-19 infections (0.73\%). The low rates of COVID-19 infections in our patients during the first wave would have also been impacted by national lockdown procedures and high levels of shielding due to our clinically vulnerable groups. Of the urological cancers, patients with prostate and renal cancers were most affected with reductions in delivery of both chemotherapy and targeted therapy respectively. This reflects clinical decision making whereby on a case-by-case basis treatment was either deferred or commenced without delay based on symptoms, growth rate of cancer, and patient factors. For instance, patients with slow growing metastatic renal cancers might have been advised to delay starting SACT for a short number of months in order to avoid hospital visits, risk of infection, and toxicities. Whereas patients diagnosed with advanced urothelial carcinoma were generally recommended to start SACT since a delay would likely lead to progressive disease over a short time period. Although there was a small increase in the number of patients receiving combination targeted/immunotherapy, this reflects the availability of axitinib/avelumab for untreated advanced renal cancers rather than an effect of the pandemic ${ }^{15}$. Reassuringly, the number of patients initiated on a new line of SACT was similar during COVID-19, albeit with fewer patients started on chemotherapy.

These differences reflect initial concerns regarding chemotherapy as a potential risk factor for severe COVID$19^{1-3}$. However, there is an increasing body of evidence that challenges this notion ${ }^{6-12}$. The current evidence does not suggest SACT as a risk factor for COVID-19, with the exception of haematological malignancies, and current clinical practice should therefore reflect this ${ }^{6-12}$. Although treatment prioritisation may explain the numbers of patients initiated on SACT, it is important to review other potential confounders. A major concern with cancer services during the pandemic is the decline in patients with cancer-related symptoms seeking medical attention during COVID-19. The number of GP appointments, 2-week waits, and core cancer diagnostic services were all significantly reduced during the first wave ${ }^{16}$. We are only starting to see some recovery in these statistics. Recent modelling studies and real-world data have suggested an increase in 'missed' cancers and shift towards higher staging ${ }^{17-19}$. Some of these patients may require SACT and therefore may partly explain the findings of our study. Another factor to consider is the availability of resources and personnel to deliver SACT due to redeployment to emergency and critical care services during COVID-19.

The decline in 6-month all-cause mortality during COVID-19 was another interesting finding. However, this figure does not take into account the proportion of patients who died from cancer and did not receive SACT due to the potential risk of contracting COVID-19 outweighing any survival benefits. There may also be an element of selection bias as the patients who were receiving SACT during COVID-19 were generally younger and of good performance status. There may also be a general decline in deaths from other nosocomial and community infectious diseases due to shielding, social distancing, and increased vigilance in infection control protocols ${ }^{20-23}$. 
It is important to appreciate the certain limitations to our study. The main limitation was that we only included patients who received SACT during COVID-19 and did not include all patients who were potentially eligible for SACT but were not given it either due to patient decision or as a result of treatment prioritisation with risk of COVID-19 infection. Therefore, we cannot comment on mortality outcomes due to this. We also only included patients with a positive COVID-19 RT-PCR test. This would have likely missed patients who were self-isolating with mild symptoms who did not get tested, death certificate diagnosis of COVID-19 in patients presenting in extremis without time for an RT-PCR test, and false negative RT-PCR results. Another limitation is the proportion of patients with incomplete data on ethnicity and performance status in the patient electronic records. This is particularly relevant as there is growing evidence that there are significant racial and socioeconomic disparities in healthcare access during COVID-19. The method of data extraction from our electronic chemotherapy prescribing system also underrepresents the number of prostate cancer patients receiving anti-gonadotropic monotherapy (e.g LHRH analgoues) as the prescriptions are continued by GPs. Finally, we do not have data regarding the provision of SACT during the second wave of COVID-19. This would be an important aspect for us to study as there were more variables to consider including availability of COVID-19 treatments, emerging safety evidence of SACT, expert consensus statements, and the arrival of novel vaccines.

\subsection{Conclusion}

This single-centre retrospective study demonstrated that patients could receive a range of SACT during COVID-19 with a low incidence of infection rate and mortality. Although shifts in the type of SACT delivered were observed with less chemotherapy administered, we were able to continue to start patients on SACT. With the emerging delta variant and rising rates of infection, we hope that our data provides reassurance that SACT can be safely delivered during a pandemic with appropriate safety provisions in place.

\subsection{Declarations}

Acknowledgements: N/A

Author's contributions: A.C.P.S and C.K. wrote the manuscript. S.D, B.R, C.M, M.J.M.I, A.C.P.S, C.K, and M.K collected the data. A.C.P.S, B.R, C.M, and M.J.M.I analysed the data. D.E, M.V.H, S.R, S.D, K.T, D.H.J, and E.P reviewed and edited the manuscript. D.E is the corresponding author.

Ethics approval and consent to participate: Consent from participants was waived as all data were collected as part of Guy's Cancer Cohort approved by our local research ethics committee at Guy's and St Thomas' NHS Hospitals (Ethics Reference number: 18/NW/0297) ${ }^{14}$. The study was performed in accordance with the Declaration of Helsinki. The research protocol for data collection and analysis was approved by Guy's and St Thomas NHS Hospitals as part of Guy's Cancer Cohort (Ethics Reference number: 18/NW/0297) ${ }^{14}$.

\section{Consent for publication: N/A}

Data availability: The datasets generated and analysed during the current study are not publicly available due to potential inferable data that may impact patient confidentiality. The reason being that our paper 
specifically refers to our institution's practices during the pandemic within a specific timeframe. Anonymising the data may impact interpretation of the analysis but is available from the corresponding author on reasonable request.

Competing interests: The authors declare no conflict of interest.

Funding information: The authors received no specific funding for this work.

\subsection{References}

1. Yu, J., Ouyang, W., Chua, MLK., Xie, C. SARS-CoV-2 Transmission in Patients With Cancer at a Tertiary Care Hospital in Wuhan, China. JAMA Oncol. 2020;6(7):1108-1110.

2. Liang, W., Guan, W., Chen, R., Wang, W., Li, J., Xu, K., Li, C., Ai, Q., Lu, W., Liang, H., Li, S., \& He, J. Cancer patients in SARS-CoV-2 infection: a nationwide analysis in China. Lancet Oncol. 2020;21(3), 335-337.

3. Dai, M., Liu, D., Liu, M., Zhou, F., Li, G., Chen, Z., et al. Patients with Cancer Appear More Vulnerable to SARS-CoV-2: A Multicenter Study during the COVID-19 Outbreak. Cancer discov. 2020;10(6), 783-791.

4. Sharafeldin, N., Bates, B., Song, Q., Madhira, V., Yan, Y., Dong, S., et al. Outcomes of COVID-19 in Patients With Cancer: Report From the National COVID Cohort Collaborative (N3C). J Clin Oncol. 2021;39(20), 2232-2246.

5. Grivas, P., Khaki, A. R., Wise-Draper, T. M., French, B., Hennessy, C., Hsu, C. Y., et al. Association of clinical factors and recent anticancer therapy with COVID-19 severity among patients with cancer: a report from the COVID-19 and Cancer Consortium. Ann Oncol. 2021;32(6), 787-800.

6. Lee, LY., Cazier, JB., Angelis, V., Arnold, R., Bisht, V., Campton, NA., et al. COVID-19 mortality in patients with cancer on chemotherapy or other anticancer treatments: a prospective cohort study. Lancet. 2020;395(10241):1919-1926.

7. Lièvre, A., Turpin, A., Ray-Coquard, I., Malicot, KL., Thariat, J., Ahle, G., et al. Risk factors for Coronavirus Disease 2019 (COVID-19) severity and mortality among solid cancer patients and impact of the disease on anticancer treatment: A French nationwide cohort study (GCO-002 CACOVID-19). Eur J Cancer. 2020;141:62-81.

8. Assaad, S., Zrounba, P., Cropet, C., ONCOVID-19 study Consortium, Blay, JY. Mortality of patients with solid and haematological cancers presenting with symptoms of COVID-19 with vs without detectable SARS-COV-2: a French nationwide prospective cohort study. Br J Cancer. 2021;1-14.

9. Baena Espinar, J., Torri, V., Whisenant, J., Hirsch, F.R., Rogado, J., de Castro Carpeño, J., et al. LBA75 Defining COVID-19 outcomes in thoracic cancer patients: TERAVOLT (Thoracic cancERs international coVid 19 cOLlaboraTion). Ann Oncol. 2020;31(Suppl 4) (abstract LBA75).

10. Jee, J., Foote, M.B., Lumish, M., Stonestrom, A.J., Wills, B., Narendra, V., et al. Chemotherapy and COVID19 Outcomes in Patients With Cancer. J Clin Oncol. 20;38(30):3538-3546 (2020).

11. Palmer, K., Sylva, R., Russell, B., Moss, C., Shah, V., Ko Ko, T., et al. Factors affecting COVID-19 outcomes in cancer patients from Guy's Cancer Center and King's College Hospital. Clin Cancer Res. 2021;27(Suppl 6) (abstract P44). 
12. Budhathoki, N., Kucharczyk, J., D'Abreo, N., Kwa, M.J., Plasilova, M., Dhage, S., et al. Risk for SARS-CoV2 infection in patients with breast cancer treated with chemotherapy, biologic therapy or active surveillance: Patient outcomes from multicenter institution in New York. J Clin Oncol. 2021;39(Suppl 15) (abstract 1513).

13. World Health Organisation. COVID-19 Clinical Management: Living guidance [Internet]. 2021. https://www.who.int/publications/i/item/WHO-2019-nCoV-clinical-2021-1. Accessed 2021 July 9.

14. Moss, C., Haire, A., Cahill, F., Enting, D., Hughes, S., Smith, D., et al. Guy's cancer cohort - real world evidence for cancer pathways. BMC Cancer. 2020;20(1):187.

15. European Medicines Agency. CHMP post-authorisation summary of positive opinion for Bavencio (II-09) [Internet]. 2019. https://www.ema.europa.eu/en/documents/smop/chmp-post-authorisation-summarypositive-opinion-bavencio-ii-09_en.pdf. Accessed 2021 July 29.

16. Cancer Research UK. Evidence of the impact of COVID-19 across the cancer pathway: Key Stats [Internet]. 2021.

https://www.cancerresearchuk.org/sites/default/files/cruk_covid_and_cancer_key_stats_june_2021.pdf. Accessed 2021 July 29

17. Maringe, C., Spicer, J., Morris, M., Purushotham, A., Nolte, E., Sullivan, R., et al. The impact of the COVID19 pandemic on cancer deaths due to delays in diagnosis in England, UK: a national, population-based, modelling study. Lancet Oncol. 2020;21:1023-1034.

18. Lai, A.G., Pasea, L., Banerjee, A., Hall, G., Denaxas, S., Chang, W.H., et al. Estimated impact of the COVID19 pandemic on cancer services and excess 1-year mortality in people with cancer and multimorbidity: near real-time data on cancer care, cancer deaths and a population-based cohort study. BMJ Open. 2020;10:e043828.

19. Vestergaard, L.S., Nielsen, J., Richter, L., Schmid, D., Bustos, N., Braeye, T., et al. Excess all-cause mortality during the COVID-19 pandemic in Europe - preliminary pooled estimates from the EuroMOMO network, March to April 2020. Euro Surveill. 2020;25(26):2001214.

20. Brueggemann, A.B., Jansen van Rensburg, M.J., Shaw, D., McCarthy, N.D., Jolley, K.A., Maiden, M.C.J., et al. Changes in the incidence of invasive disease due to Streptococcus pneumoniae, Haemophilus influenzae, and Neisseria meningitidis during the COVID-19 pandemic in 26 countries and territories in the Invasive Respiratory Infection Surveillance Initiative: a prospective analysis of surveillance data. Lancet Digit Health. 2021;3(6):e360-e370.

21. Losurdo, P., Paiano, L., Samardzic, N., Germani, P., Bernardi, L., Borelli, M., et al. Impact of lockdown for SARS-CoV-2 (COVID-19) on surgical site infection rates: a monocentric observational cohort study. Updates Surg. 2020;72(4):1263-1271.

22. Cerulli Irelli, E., Orlando, B., Cocchi, E., Morano, A., Fattapposta, F., Di Piero, V., et al. The potential impact of enhanced hygienic measures during the COVID-19 outbreak on hospital-acquired infections: $A$ pragmatic study in neurological units. J Neurol Sci. 2020;15;418:117111.

23. Ponce-Alonso, M., Sáez de la Fuente, J., Rincón-Carlavilla, A., Moreno-Nunez, P., Martínez-García, L., Escudero-Sánchez, R., et al. Impact of the coronavirus disease 2019 (COVID-19) pandemic on nosocomial Clostridioides difficile infection. Infect Control Hosp Epidemiol. 2021;42(4):406-410. 


\section{Supplementary Files}

This is a list of supplementary files associated with this preprint. Click to download.

- UrologySACTSupplementaryFinal.docx 